Special Issue of the 7th International Advances in Applied Physics and Materials Science (APMAS 2017)

\title{
Resistive Switching Behaviour in PMMA/Al:ZnO Composite Films
}

\author{
S. Vallabhapurapu ${ }^{a, b, *}$, L.D. Varma Sangani ${ }^{c}$, M. Ghanashyam Krishna ${ }^{c}$, J. Das $^{d}$, \\ C. $\mathrm{TU}^{b}, \mathrm{~S} . \mathrm{DU}^{e}$ AND A. SRINIVASAN ${ }^{f, g}$ \\ ${ }^{a}$ School of Computing, University of South Africa, Johannesburg 1710, South Africa \\ ${ }^{b}$ Computer Systems Engineering, Faculty of Information and Communication Technology, \\ Tshwane University of Technology, Pretoria 0001, South Africa \\ ${ }^{c}$ CASEST, School of Physics, University of Hyderabad, Hyderabad 500046, India \\ ${ }^{d}$ Silicon Institute of Technology, Bhubaneswar 751024, India \\ ${ }^{e}$ Department of Electrical Engineering, Faculty of Engineering and Built Environment, \\ Tshwane University of Technology, Pretoria 0001, South Africa \\ ${ }^{f}$ Department of Physics, CSET, University of South Africa, Johannesburg 1710, South Africa \\ ${ }^{g}$ Department of Physics, Indian Institute of Technology, Guwahati 781039, India
}

\begin{abstract}
Considering the potential of polymer nanocomposites in resistive memory application, electrical conduction in poly methyl methacrylate thin films embedded with $\mathrm{Al}$ doped $\mathrm{ZnO}$ nanoparticles has been investigated. This polymer nanocomposite was spin coated on indium tin oxide coated glass plate and a titanium film was coated on top of this to form a device. Current-voltage characteristics of the fabricated device was measured. The current increased gradually with the increase of voltage, reaching a peak and dropped down to the low current (OFF) state. Upon further increase of voltage, a sharp increase of current (three orders of magnitude) or switching from low current $(\mathrm{OFF})$ state to high current $(\mathrm{ON})$ state occurred at $\sim 2.9 \mathrm{~V}$. Current-voltage characteristics have shown Ohmic behaviour in the low voltage regime followed by hopping type of conductivity prior to switching.
\end{abstract}

DOI: 10.12693/APhysPolA.134.68

PACS/topics: PMMA nano composite, Al:ZnO nanoparticle, electrical conduction, resistive switching

\section{Introduction}

The memory phenomenon in nanoparticle-polymer composites which is essentially based on two distinct electrical states commonly known as the $\mathrm{ON}$ and $\mathrm{OFF}$ states [1-11], is attracting immense attention. The excitement in these polymer nanocomposites stems from the zeal to understand the conduction mechanism and more importantly the possibility of developing improved memory systems as compared to the existing ones. The improvements possible include flexibility, biodegradability, simple fabrication and processing, and cost reduction.

Metal and semiconducting nano particles such as CdSe, $\mathrm{ZnO}$, gold and silver are often used in a variety of polymer matrices to achieve resistive switching based memory effects. A voltage bias usually drives the nano particlepolymer (NP-P) composite into an electrical bistability or switching states. Depending on the sequence of bias application, the system is driven into either a low resistance state (LRS) or the high resistance state (HRS). However, these switching characteristics are strongly dependent on the polymer, the nano particles, their concentrations, the electrodes, etc.

*corresponding author; e-mail: vallas@unisa.ac.za
Spun cast metal or semiconducting nano particle embedded poly methyl methacrylate (PMMA) films are promising candidates to achieve reasonably good bistability and memory performance, such as high $\mathrm{ON} / \mathrm{OFF}$ ratio, retention time, and endurance, as indicated by a few reports in the literature [12-16].

Bistability can be achieved in $\mathrm{O}, \mathrm{N}$ or S-shaped current-voltage $(I-V)$ characteristics [17]. $\mathrm{N}$ and Sshaped $I-V$ characteristics exhibit a sudden jump in current at a threshold voltage value, whereas O-shaped characteristic is devoid of such an abrupt jump in current but rather involves a cyclic variation in current. Most interestingly, reports in the literature show that not all nano particle embedded PMMA composites show the same kind of bistability. In some cases it is O-shaped and in the others it is S-shaped bistability. For example, in the case of $\mathrm{ZnO}$ embedded PMMA [12] and PMMA-Ag nano particle bilayer composite films [14], O-shaped bistability has been reported. On the other hand, in PMMA/Graphene/PMMA [13] and $\mathrm{Al} / \mathrm{ZnO}$ embedded PMMA/ITO/PET [15], S-shaped bistability has been observed.

To complicate matters, the conduction mechanisms in each of these systems are quite different. Thus, based on reports of different types of bistability and conduction mechanisms in PMMA embedded with different nanoparticles, PMMA nanocomposites appear to be a very interesting system to study regarding the conduction mech- 
anism and associated memory behaviour. In this paper, we report fabrication of spun cast $\mathrm{Al}_{0.02} \mathrm{Zn}_{0.98} \mathrm{O}$ nano particle embedded PMMA thin films and their memory behaviour.

\section{Experimental}

$\mathrm{Al}_{0.02} \mathrm{Zn}_{0.98} \mathrm{O}$ nanoparticles with wurtzite structure and average crystallite size of $30-50 \mathrm{~nm}$ were first prepared by chemical pyrophoric method described elsewhere [18]. $\quad 0.5 \mathrm{w} / \mathrm{v} \%$ PMMA (MW=4000, SigmaAldrich) solution was prepared in toluene with continuous stirring for one hour. $0.5 \mathrm{w} / \mathrm{v} \% \mathrm{Al}_{0.02} \mathrm{Zn}_{0.98} \mathrm{O}$ nanoparticles were dispersed in the PMMA solution and ultrasonicated for 15 minutes until a clear solution was obtained. The $\mathrm{w} / \mathrm{v} \%$ error bar is only $0.001 \%$.

This solution was spin coated on pre-cleaned indium tin oxide (ITO) coated glass substrate (Sigma-Aldrich) at a speed of $1200 \mathrm{rpm}$ for 35 seconds. The film thickness was measured to be $1.97 \mu \mathrm{m}$ using an atomic force microscope (Nanosurf). The highly transparent films were then dried at $60{ }^{\circ} \mathrm{C}$ in air.

Titanium electrodes with thickness of $200 \mathrm{~nm}$ were sputter deposited on the films using $2 \mathrm{~mm}$ shadow mask. $I-V$ characteristics of the fabricated device were recorded using Agilent B1500 semiconductor parameter analyser at room temperature in the range 0 to $3.5 \mathrm{~V}$. The measurement has the accuracy up to $1 \mathrm{pA}$ for current and $5 \mu \mathrm{V}$ for voltage.

\section{Results and discussion}

Figure 1 shows the structure of the fabricated device. Spin coated PMMA/ $\mathrm{Al}_{0.02} \mathrm{Zn0} 0 .{ }_{98} \mathrm{O}$ nanoparticulate film is sandwiched between the ITO and Ti electrodes. The biasing scheme used in the measurement is also shown in the figure.

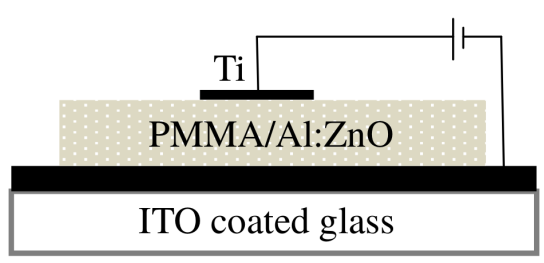

Fig. 1. Structure of the fabricated device with $\mathrm{PMMA} / \mathrm{Al}: \mathrm{ZnO}$ film as the active layer.

Figure 2 shows the $I-V$ characteristics of the device. It is evident from the $I-V$ characteristics, that the device exhibits S-type bistability. In order to understand the conduction mechanisms prior to the electrical switching, an enlarged view of the $I-V$ characteristics in the $0 \mathrm{~V}$ to $2.9 \mathrm{~V}$ range is shown as an inset in Fig. 2. There is a linear rise in current in the voltage range from $0 \mathrm{~V}$ to $0.5 \mathrm{~V}$ followed by an exponential increase till $2.26 \mathrm{~V}$. The two regions are clearly shown in the figure. The current then drops and the device makes a transition to the high resistance state (HRS) or OFF state till the voltage is $2.9 \mathrm{~V}$.

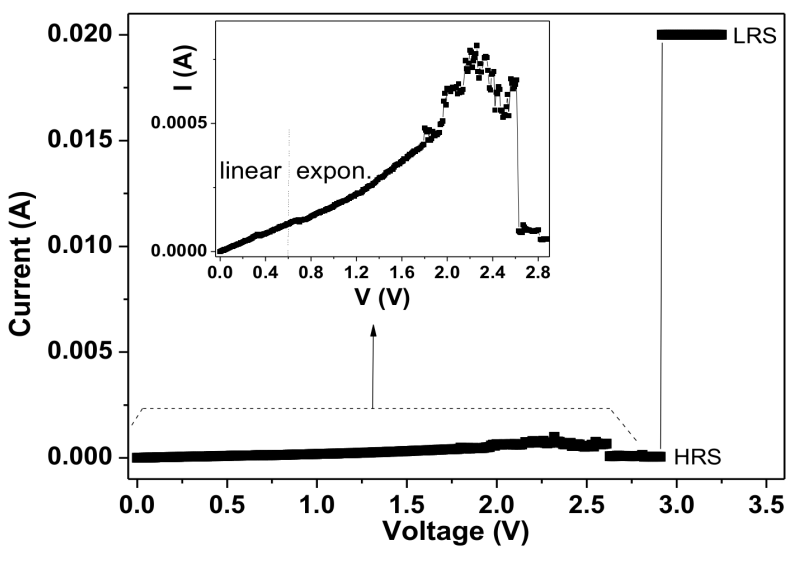

Fig. 2. $I-V$ characteristics and switching behaviour of PMMA/Al:ZnO device. Inset shows the $I-V$ data from 0 to $2.9 \mathrm{~V}$.

In the $0 \mathrm{~V}$ to $0.5 \mathrm{~V}$ region, the $I-V$ data fits well to a linear relation indicating an Ohmic conduction behaviour. Between $0.5 \mathrm{~V}$ to $2.26 \mathrm{~V}$, the data fits well to an exponential function of the type [19],

$$
I=I_{0}+A \exp \left\{\left(V-V_{\mathrm{a}}\right) / k T\right\} .
$$

In Eq. (1), $I_{0}$ is residual current (if any), the constant $A$ is a product of mean hopping distance, number of electrons and thermal vibrational frequency of electrons at the trap, $e V_{\mathrm{a}}$ is the activation energy (where $e$ is the electron charge) and $k$ is the Boltzmann constant.

Figure 3 shows the exponential fit for the voltage sweep between $0.5 \mathrm{~V}$ to $2.26 \mathrm{~V}$ and the inset in Fig. 3 shows the linear fit in the region of $0-0.5 \mathrm{~V}$. The exponential fit in the region $0.5 \mathrm{~V}$ to $2.26 \mathrm{~V}$ indicates a hopping type conductivity mechanism [19]. Such hopping behaviour is well known in the PMMA system due to the presence of polar groups in PMMA [20].

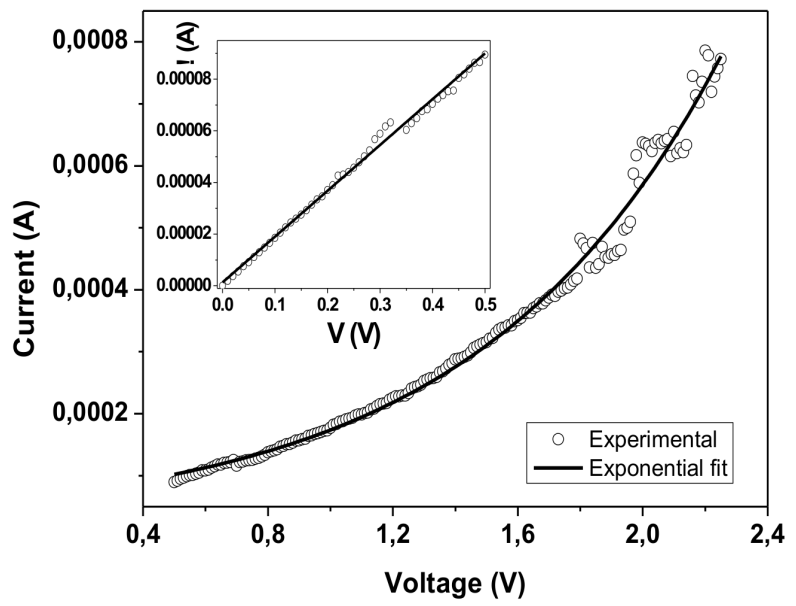

Fig. 3. Exponential fit to $I-V$ data from 0.5 to $2.26 \mathrm{~V}$. Inset shows linear fit to $I-V$ data from 0 to $0.5 \mathrm{~V}$.

Around the peak in current at $2.26 \mathrm{~V}$ there is some kind of frustration in the system, as evidenced by fluctu- 
ations in current which are followed by a drop to low current state (HRS). One should also note that this drop is not a sharp one and cannot be associated with switching mechanism although some polymer composites exhibit pre-switching and multiple stage switching behaviours [21, 22].

These two conduction mechanisms, namely, the Ohmic conduction followed by electron hopping lead to the "setting" of the device in the HRS prior to switching at $2.9 \mathrm{~V}$. Once this threshold voltage is reached, the current jumps by more than three orders of magnitude to the low resistance state (LRS) or the ON state with typical S-type switching characteristics. The current remains constant beyond this voltage since it reaches the compliance value of $20 \mathrm{~mA}$ and further increase would damage the device.

It should be noted that the resistive switching behaviour observed in this study is different from the Oshaped bistability reported by Pradhan, et al. [12] in $\mathrm{ZnO}$ nanoparticle embedded PMMA spin coated thin films. The conduction mechanism involved in their case is of thermionic emission type for both the low and high resistance states.

The reported conduction mechanisms observed by Son, et al. [15], viz., thermionic emission below $1 \mathrm{~V}$ and space charge limited conduction in the range $1 \mathrm{~V}$ to $3.6 \mathrm{~V}$, are again completely different from our case. However, Sshaped resistive switching has been reported in $\mathrm{Al} / \mathrm{ZnO}$ nano particle embedded PMMA/ITO on flexible PET by Son, et al. [15], where the resistive switching occurs at a threshold voltage of $4 \mathrm{~V}$ which is far higher than our observation.

A comparison of our results with those in the literature reveals that the conduction mechanism and the switching characteristics vary even in devices fabricated with PMMA as the dispersant and with similar type of filler, say $\mathrm{ZnO}$. Hence, the observed differences seem to have stemmed from the concentration of the filler and processing conditions, leading to differences in film thickness and distribution of filler particles. By careful control of these factors, it might be possible to obtain different conduction mechanisms and switching characteristics in the same polymer-nanoparticle film.

\section{Conclusions}

In conclusion, we report S-type resistive switching behaviour in $\mathrm{Al}: \mathrm{ZnO}$ nano particle embedded PMMA spin coated micron sized thin films. Stable switching from HRS to LRS was observed in the fabricated device at $2.9 \mathrm{~V}$, which is lower than that reported in the literature for a similar device. OFF/ON ratio of more than three orders of current jump exhibited by the device shows clear distinction between the two electrical states. The conduction mechanisms leading to the "setting" of the HRS prior to switching at $2.9 \mathrm{~V}$ were analysed. We found Ohmic type of conduction at low voltages followed by hopping type of conduction which leads to frustration and setting up of the HRS prior to S-type bistable switching in the device.
While the three orders of current change between the HRS and LRS at $\sim 2.9 \mathrm{~V}$ is encouraging in terms of switching voltage as compared to the reports on $\mathrm{ZnO}$ embedded PMMA, the endurance stability needs to be understood and further optimization is required to obtain a resistive random access memory cell using such a device.

\section{References}

[1] B. Cho, S. Song, Y. Ji, T.-W. Kim, T. Lee, Adv. Funct. Mater. 21, 2806 (2011).

[2] Y. Sun, D. Wen, X. Bai, Phys. Chem. Chem. Phys. 20, 5771 (2018).

[3] Y. Yang, J. Ouyang, L. Ma, R.J.-H. Tseng, C.-W.Chu, Adv. Funct. Mater. 16, 1001 (2006).

[4] A. Kathalingam, J.-K. Rhee, J. Electron. Mater. 41, 2162 (2012).

[5] W.P. Lin, S.J. Liu, T. Gong, Q. Zhao, W. Huang, Adv. Mater. 26, 570 (2014).

[6] Y.-C. Chang, Y.-H. Wang, Appl. Phys. Lett. 106 123302 (2015).

[7] D. Ma, M. Aguiar, J.A. Freire, I.A. Huemmelgen, Adv. Mater. 12, 1063 (2000).

[8] Q.-D. Ling, D.-J. Liaw, E.Y.-H. Teo, C. Zhu, D.S.H. Chan, E.-T. Kang, K.-G. Neoh, Polymer 48, 5182 (2007).

[9] K. Onlaor, N. Chaithanatkun, B. Tunhoo, Curr. Appl. Phys. 16, 1418 (2016).

[10] S. Valanarasu, A. Kathalingam, J.-K. Rhee, R. Chandramohan, T.A. Vijayan, M. Karunakaran, J. Nanosci. Nanotechnology 15, 1416 (2015).

[11] N.R. Hosseini, J.-S. Lee, Adv. Funct. Mater. 25, 5586 (2015).

[12] B. Pradhan, S.K. Majee, S.K. Batabyal, A.J. Pal, J. Nanosci. Nanotechnol. 7, 4534 (2007).

[13] D.-I. Son, T.W. Kim, T.H. Shim, J.H. Jung, D.U. Lee, J.M. Lee, W.I. Park, W.K. Choi, Nanolett. 10, 2441 (2010).

[14] B. Mukharjee, M. Mukherjee, Appl. Phys. Lett. 94, 173510 (2009).

[15] D.-I. Son, D.-H. Park, W.K. Choi, S.-H. Cho, W.T. Kim, T.W. Kim, Nanotechnology 20, 195203 (2009).

[16] Y. Zhou, S.-T. Han, Z.-X. Xu, V.A.L. Roy, Nanotechnology 23, 344014 (2012).

[17] D. Prime, S. Paul, Phil. Trans. R. Soc. A 367, 4141 (2009).

[18] J. Das, D.K. Mishra, V.V. Srinivasu, J. Alloys Compds. 704, 237 (2017).

[19] F.-C. Chiu, Adv. Mater. Sci. Eng. 2014, 578168 (2014).

[20] K.S. Suh, H.J. Lee, C.G. Kang, IEEE Trans. Dielectr. Electr. Insul. 2, 460 (1995).

[21] Y.-C. Chang, Y.-H. Wang, ACS Appl. Mater. Interfaces 6, 5413 (2014).

[22] F. Pan, S. Gao, C. Chen, C. Song, F. Zeng, Mater. Sci. Eng. R 83, 1 (2014). 